\title{
Solution of Singular Integral Equations Involving Logarithmically Singular Kernel with an Application in a Water Wave Problem
}

\author{
Sudeshna Banerjea, ${ }^{1}$ Barnali Dutta, ${ }^{2}$ and A. Chakrabarti ${ }^{3}$ \\ ${ }^{1}$ Department of Mathematics, Jadavpur University, Kolkata 700032, India \\ ${ }^{2}$ Department of Mathematics, Netajinagar Day College, Regent Estate, NSC Bose Road, \\ Kolkata 700023, India \\ ${ }^{3}$ Department of Mathematics, Indian Institute of Science, Bangalore 560012, India
}

Correspondence should be addressed to Sudeshna Banerjea, sbanerjee@math.jdvu.ac.in

Received 10 March 2011; Accepted 1 April 2011

Academic Editors: A. Bellouquid and T. Y. Kam

Copyright (C) 2011 Sudeshna Banerjea et al. This is an open access article distributed under the Creative Commons Attribution License, which permits unrestricted use, distribution, and reproduction in any medium, provided the original work is properly cited.

A direct function theoretic method is employed to solve certain weakly singular integral equations arising in the study of scattering of surface water waves by vertical barriers with gaps. Such integral equations possess logarithmically singular kernel, and a direct function theoretic method is shown to produce their solutions involving singular integrals of similar types instead of the stronger Cauchy-type singular integrals used by previous workers. Two specific ranges of integration are examined in detail, which involve the following: Case(i) two disjoint finite intervals $(0, a) \cup(b, c)$ and ( $a, b, c$ being finite) and Case(ii) a finite union of $n$ disjoint intervals. The connection of such integral equations for Case(i), with a particular water wave scattering problem, is explained clearly, and the important quantities of practical interest (the reflection and transmission coefficients) are determined numerically by using the solution of the associated weakly singular integral equation.

\section{Introduction}

The singular integral equation

$$
\int_{B} \phi(t) \ln \left|\frac{t-x}{t+x}\right| d t=f(x), \quad x \in B
$$

where $B$ is a single or a finite union of finite intervals is encountered in many branches of mathematical physics (cf. Lewin [1], Ursell [2], and Mandal and Kundu [3]).This type 
of integral equation is usually solved in the literature by converting the weak singularity (logarithmic type) in the kernel to a Cauchy-type singularity which is strong in nature. For an integral equation with weakly singular kernel, the associated integral is defined in the standard Riemann sense, while for an integral equation with strongly singular kernel, the singular integral has to be defined appropriately so as to give a consistent mathematical sense. Consequently, the integrals with weak singularities are more amenable to the numerical techniques as compared to the integrals with strong singularity which requires artificial methods for its numerical evaluation. Noting this difficulty with the integrals with strong singularities, Chakrabarti and Manam [4], Manam [5], Banerjea and Dutta [6], and Banerjea and Sarkar [7] used function theoretic method to solve (1.1) for various $B$ without converting it to Cauchy-type integral equation. However, the solution function in [4-7] involves Cauchytype singularity.

Recently, Chakrabarti [8] has developed a direct function theoretic method for (1.1) with $B=[a, b]$ to obtain its solution. The novelty of the method is that it avoids the occurrence of strong singularity such as Cauchy-type singularity in the solution function of the integral equation.

The present paper is a generalization of the method in [8] with a little modification. We have applied the method of [8] to the integral equation (1.1), where $B$ consists of (i) two disjoint finite intervals $(0, a) \cup(b, c)$ and (ii) a finite union of $n$ disjoint intervals $\cup_{i=1}^{n}\left(a_{i}, b_{i}\right)$. As an application, we have applied the solution of (1.1) where $B$ consists of two disjoint finite intervals $(0, a) \cup(b, c)$ to the problem of water wave scattering by a partially immersed vertical barrier with a gap present in deep water. The important quantities of physical interest, that is, the reflection and transmission coefficients, are evaluated. The numerical values of the reflection coefficient are presented graphically for various values of the wave number and various lengths of the barrier. It is observed that for certain small range of small values of wave number, there occurs almost total reflection. This phenomenon was also observed by Banerjea [9]. It is also observed that this phenomenon of almost total reflection of waves decreases when the length of lower part of the barrier increases and also when the length of the gap increases. We observe that by letting $a \rightarrow 0$, the known results available in the literature are recovered. It is emphasized here that for numerical evaluation of the reflection and transmission coefficients, computation of singular integrals with strong singularities is avoided, and this is the main advantage in the present method over other methods existing in the literature.

In Section 2, we have presented the detailed analysis to determine the solution of the integral equation (1.1) in cases (i) and (ii) mentioned in the abstract. Then, in Section 3, we have shown the connection of (1.1) with a water wave scattering problem.

\section{Solution of the Integral Equation (1.1)}

\subsection{Case(i)}

Consider that

$$
\mathbf{B}=(0, a) \cup(b, c)
$$


In this section, we present a method of solution of

$$
\int_{B} \phi(t) \ln \left|\frac{t-x}{t+x}\right| d t=f(x), \quad x \in B
$$

with $B \equiv(0, a) \cup(b, c)$ and

$$
\phi(t) \sim O(|t-p|)^{-1 / 2} \text { as } t \longrightarrow p
$$

$p$ being $a, b$, or $c$.

We introduce a sectionally analytic function $F(z)$ in the complex $z(=x+i y)$ plane, cut along the segment $(-b,-c) \cup(-a, 0) \cup(0, a) \cup(b, c)$ on the real axis as given by

$$
F(z)=\int_{B} \phi(t) \ln \left(\frac{t-z}{t+z}\right) d t, \quad(z=x+i y, x \in B)
$$

If we use the following results, valid for the limiting values of $\Phi(z)=\log (z) \equiv \log (x+i y)$ :

$$
\Phi^{ \pm}(x) \sim \begin{cases}\ln |x| & \text { for } x>0 \\ \ln |x| \pm \pi i & \text { for } x<0\end{cases}
$$

we find the following limiting values of $F(z)$ :

$$
F^{ \pm}(x)= \begin{cases}\int_{B} \phi(t) \ln \left|\frac{t-x}{t+x}\right| d t \mp \pi i \int_{0}^{x} \phi(t) d t, & x \in(0, a), \\ \int_{B} \phi(t) \ln \left|\frac{t-x}{t+x}\right| d t \mp \pi i \int_{0}^{a} \phi(t) d t \mp \pi i \int_{b}^{x} \phi(t) d t, & x \in(b, c), \\ \int_{B} \phi(t) \ln \left|\frac{t-x}{t+x}\right| d t \pm \pi i \int_{0}^{-x} \phi(t) d t, & x \in(-a, 0), \\ \int_{B} \phi(t) \ln \left|\frac{t-x}{t+x}\right| d t \pm \pi i \int_{0}^{-x} \phi(t) d t, & x \in(-c,-b) .\end{cases}
$$

Equations (2.6) give rise to the following Plemelj-type alternative formulae:

$$
F^{+}(x)-F^{-}(x)=r(x)
$$


where

$$
r(x)= \begin{cases}-2 \pi i \int_{0}^{x} \phi(t) d t, & x \in(0, a), \\ -2 \pi i \int_{0}^{a} \phi(t) d t-2 \pi i \int_{b}^{x} \phi(t) d t, & x \in(b, c), \\ 2 \pi i \int_{0}^{-x} \phi(t) d t, & x \in(-a, 0), \\ 2 \pi i \int_{0}^{a} \phi(t) d t+2 \pi i \int_{b}^{-x} \phi(t) d t, & x \in(-c,-b), \\ F^{+}(x)+F^{-}(x)=2 \int_{B} \phi(t) \ln \left|\frac{t-x}{t+x}\right| d t, & x \in\left(B \cup B^{\prime}\right),\end{cases}
$$

where $B^{\prime}=(-c,-b) \cup(-a, 0)$.

By using the relation (2.9), the singular integral equation (2.2) can be expressed in the form of a Riemann-Hilbert problem as given by

$$
F^{+}(x)+F^{-}(x)=2 h(x), \quad x \in\left(B \cup B^{\prime}\right),
$$

where

$$
h(x)= \begin{cases}f(x), & x \in B \\ -f(-x), & x \in B^{\prime}\end{cases}
$$

Again noting the relation (2.8), we can write

$$
r^{\prime}(x)= \begin{cases}-2 \pi i \phi(x), & x \in B \\ -2 \pi i \phi(-x), & x \in B^{\prime}\end{cases}
$$

Hence,

$$
\phi(x)=-\frac{1}{2 \pi i} r^{\prime}(x), \quad x \in B .
$$

Using the relation (2.13) in (2.4), we get the solution of special Riemann-Hilbert problem (2.7) as

$$
F(z)=-\frac{1}{2 \pi i} \int_{B} r^{\prime}(t) \ln \left(\frac{t-z}{t+z}\right) d t
$$


Utilizing the above idea, we can solve the Riemann-Hilbert problem (2.10) in the following manner.

Let

$$
F(z)=F_{0}(z) F_{2}(z)
$$

where $F_{0}(z)$ is the solution of the corresponding homogeneous problem (2.10), that is,

$$
F_{0}^{+}(x)+F_{0}^{-}(x)=0
$$

Thus, (2.10) becomes

$$
F_{2}^{+}(x)-F_{2}^{-}(x)=\frac{2 h(x)}{F_{0}^{+}(x)}, \quad x \in B \cup B^{\prime}
$$

This is a Riemann-Hilbert problem which is similar to (2.7). Noting (2.7), (2.12), and (2.14), the solution of this Riemann-Hilbert problem is given by

$$
F_{2}(z)=\frac{F(z)}{F_{0}(z)}=-\frac{1}{2 \pi i} \int_{B}\left(\left(\frac{d}{d t} \frac{2 f(t)}{F_{0}^{+}(t)}\right) \ln \left(\frac{t-z}{t+z}\right)\right) d t+E(z)
$$

where $E(z)$ is an entire function.

We can choose

$$
F_{0}(z)=\left[z^{2}\left(z^{2}-a^{2}\right)\left(z^{2}-b^{2}\right)\left(z^{2}-c^{2}\right)\right]^{-1 / 2}
$$

with

$$
F_{0}^{ \pm}(x)= \begin{cases} \pm \frac{i}{\rho(x)}, & x \in(0, a) \\ \mp \frac{i}{\rho(x)}, & x \in(b, c)\end{cases}
$$

where

$$
\rho(x)=\left|x^{2}\left(x^{2}-a^{2}\right)\left(x^{2}-b^{2}\right)\left(x^{2}-c^{2}\right)\right|^{1 / 2} .
$$


Knowing $F(z)$ from (2.18), (2.19), and (2.20), we can find the limiting values $F^{ \pm}(x)$ of $F(z)$ using the formula (2.5). Substituting $F^{ \pm}(x)$ in (2.7) and noting the relation (2.8), the solution of the integral equation (2.2) can be obtained as

$$
\phi(x)= \begin{cases}\frac{d}{d x}\left(\frac{\psi(x)}{\rho(x)}\right), & x \in(0, a), \\ -\frac{d}{d x}\left(\frac{\psi(x)}{\rho(x)}\right), & x \in(b, c),\end{cases}
$$

where

$$
\begin{aligned}
\psi(x)= & -\frac{1}{\pi^{2}} \int_{0}^{a} \frac{d}{d t}(f(t) \rho(t)) \ln \left|\frac{t-x}{t+x}\right| d t+\frac{1}{\pi^{2}} \int_{b}^{c} \frac{d}{d t}(f(t) \rho(t)) \ln \left|\frac{t-x}{t+x}\right| d t \\
& -\pi\left(A x^{4}+B x^{3}+C x^{2}+D x+E\right),
\end{aligned}
$$

and $A, B, C, D$, and $E$ are arbitrary constants.

It is observed here that the original integral equation (2.2) can be solved by the aid of formula (2.22) if and only if the following consistency conditions are satisfied:
(i) $\psi(0)=0$,
(ii) $\psi^{\prime}(0)=0$,
(iii) $\psi(a)=0$,
(iv) $\psi(b)=0$,
(v) $\psi(c)=0$.

We can determine the arbitrary constants $A, B, C, D$, and $E$ by using the relation (2.24). Thus, $\phi(x)$ can be determined completely by utilizing the relation (2.22). It is observed that the solution as obtained by the relations (2.22) and (2.23) of (2.2) neither involves any strong singularity nor any arbitrary constant.

\subsection{Case(ii)}

Consider that

$$
\mathbf{B}=B_{1}=\cup_{i=1}^{n}\left(a_{i}, b_{i}\right)
$$

In this section, we proceed to solve the integral equation

$$
\int_{B_{1}} \phi_{1}(t) \ln \left|\frac{t-x}{t+x}\right| d t=f_{1}(x), \quad x \in B_{1}
$$

where $B_{1}$ consists of $n$ finite disjoint intervals $B_{1}=\cup_{i=1}^{n}\left(a_{i}, b_{i}\right)$ and $\phi_{1}(t) \sim O\left(\left|t-p_{i}\right|\right)^{-1 / 2}$ as $t \rightarrow p_{i}, p_{i}$ being $a_{i}, b_{i}, i=1, \ldots, n$. 
In this case, we introduce the following sectionally analytic function

$$
F_{1}(z)=\int_{B_{1}} \phi_{1}(t) \ln \left(\frac{t-z}{t+z}\right) d t, \quad\left(z=x+i y, x \in B_{1}\right)
$$

cut along $\cup_{i=1}^{n}\left(-b_{i},-a_{i}\right) \cup_{i=1}^{n}\left(a_{i}, b_{i}\right)$.

Using the formula (2.5), we get following limiting values of $F(z)$ :

$$
\begin{aligned}
& F_{1}^{ \pm}(x) \\
& = \begin{cases}\int_{B_{1}} \phi_{1}(t) \ln \left|\frac{t-x}{t+x}\right| d t \mp \pi i \int_{a_{1}}^{x} \phi_{1}(t) d t, & x \in\left(a_{1}, b_{1}\right), \\
\int_{B_{1}} \phi_{1}(t) \ln \left|\frac{t-x}{t+x}\right| d t \mp \pi i \int_{a_{1}}^{b_{1}} \phi_{1}(t) d t \mp \pi i \int_{a_{2}}^{x} \phi_{1}(t) d t, & x \in\left(a_{2}, b_{2}\right), \\
\int_{B_{1}} \phi_{1}(t) \ln \left|\frac{t-x}{t+x}\right| d t \mp \pi i \int_{a_{1}}^{b_{1}} \phi_{1}(t) d t \mp \pi i \int_{a_{n-1}}^{b_{n-1}} \phi_{1}(t) d t \mp \pi i \int_{a_{n}}^{x} \phi_{1}(t) d t, & x \in\left(a_{n}, b_{n}\right), \\
\int_{B_{1}} \phi_{1}(t) \ln \left|\frac{t-x}{t+x}\right| d t \pm \pi i \int_{a_{1}}^{-x} \phi_{1}(t) d t, & x \in\left(-b_{1},-a_{1},\right), \\
\int_{B_{1}} \phi_{1}(t) \ln \left|\frac{t-x}{t+x}\right| d t \pm \pi i \int_{a_{1}}^{b_{1}} \phi_{1}(t) d t \pm \pi i \int_{a_{2}}^{-x} \phi_{1}(t) d t, & x \in\left(-b_{2},-a_{2}\right), \\
\int_{B_{1}} \phi_{1}(t) \ln \left|\frac{t-x}{t+x}\right| d t \pm \pi i \int_{a_{1}}^{b_{1}} \phi_{1}(t) d t \pm \pi i \int_{a_{n-1}}^{b_{n-1}} \phi_{1}(t) d t \pm \pi i \int_{a_{n}}^{x} \phi_{1}(t) d t, & x \in\left(-b_{n},-a_{n}\right) .\end{cases}
\end{aligned}
$$

This gives the following Plemelj-type alternative formulae for the integral equation (2.26):

$$
F_{1}^{+}(x)-F_{1}^{-}(x)=r_{1}(x)
$$

where

$$
r_{1}(x)= \begin{cases}-2 \pi i \int_{a_{1}}^{x} \phi_{1}(t) d t, & x \in\left(a_{1}, b_{1}\right), \\ -2 \pi i \int_{a_{1}}^{b_{1}} \phi_{1}(t) d t-2 \pi i \int_{a_{2}}^{x} \phi_{1}(t) d t, & x \in\left(a_{2}, b_{2}\right), \\ -2 \pi i \bigcup_{j=1}^{(n-1)} \int_{a_{j}}^{b_{j}} \phi_{1}(t) d t-2 \pi i \int_{a_{n}}^{x} \phi_{1}(t) d t, & x \in\left(a_{n}, b_{n}\right), \\ 2 \pi i \int_{a_{1}}^{-x} \phi_{1}(t) d t, & x \in\left(-b_{1},-a_{1}\right), \\ 2 \pi i \int_{a_{1}}^{b_{1}} \phi_{1}(t) d t+2 \pi i \int_{a_{2}}^{-x} \phi_{1}(t) d t, & x \in\left(-b_{2},-a_{2}\right), \\ 2 \pi i \bigcup_{j=1}^{(n-1)} \int_{a_{j}}^{b_{j}} \phi_{1}(t) d t-2 \pi i \int_{a_{n}}^{-x} \phi_{1}(t) d t, & x \in\left(-b_{n},-a_{n}\right),\end{cases}
$$




$$
F_{1}^{+}(x)+F_{1}^{-}(x)= \begin{cases}2 f_{1}(x), & x \in B_{1}, \\ -2 f_{1}(-x), & x \in B_{1}^{\prime} \equiv \cup_{i=1}^{n}\left(-b_{i},-a_{i}\right) .\end{cases}
$$

Hence, from the relation (2.30), we get

$$
\phi_{1}(x)=-\frac{1}{2 \pi i} r_{1}^{\prime}(x), \quad x \in B_{1} .
$$

Thus, noting the relation (2.32), the equation (2.27) becomes

$$
F_{1}(z)=-\frac{1}{2 \pi i} \int_{B_{1}} r_{1}^{\prime}(t) \ln \left(\frac{t-z}{t+z}\right) d t
$$

Utilizing the above idea, we can solve the Riemann-Hilbert problem (2.31) in the following form:

$$
\frac{F_{1}(z)}{F_{1}^{(0)}(z)}=-\frac{1}{2 \pi i} \int_{B_{1}}\left(\left(\frac{d}{d t} \frac{2 f_{1}(t)}{F_{1}^{(0)+}(t)}\right) \ln \left(\frac{t-z}{t+z}\right)\right) d t+E_{1}(z)
$$

where $E_{1}(z)$ is an entire function, and we can choose $F_{1}^{(0)}(z)$, the solution of the homogeneous equation of (2.31) as

$$
F_{1}^{(0)}(z)=\prod_{i=1}^{n}\left[\left(z^{2}-a_{i}^{2}\right)\left(z^{2}-b_{i}^{2}\right)\right]^{-1 / 2},
$$

with

$$
F_{1}^{(0) \pm}(x)= \begin{cases} \pm \frac{(-1)^{n} i}{\rho_{1}(x)}, & x \in\left(a_{1}, b_{1}\right), \\ \mp \frac{(-1)^{n} i}{\rho_{1}(x)}, & x \in\left(a_{2}, b_{2}\right), \\ \pm \frac{(-1)^{n} i}{\rho_{1}(x)}, & x \in\left(a_{3}, b_{3}\right), \\ \vdots & \\ \mp \frac{i}{\rho_{1}(x)}, & x \in\left(a_{n}, b_{n}\right),\end{cases}
$$

where

$$
\rho_{1}(x)=\prod_{i=1}^{n}\left[\left(x^{2}-a_{i}^{2}\right)\left(x^{2}-b_{i}^{2}\right)\right]^{1 / 2} .
$$


Using (2.35) in (2.34), $F_{1}(z)$ is known. Noting the relation (2.5), one can find the limiting values $F_{1}^{ \pm}(x)$ of $F_{1}(z)$. Using the limiting values $F_{1}^{ \pm}(x)$, we obtain, from (2.29) and (2.30), the solution $\phi_{1}(x)$ of the integral equation (2.26) as

$$
\phi_{1}(x)= \begin{cases}\frac{d}{d x}\left(\frac{\psi_{1}(x)}{\rho_{1}(x)}\right), & x \in\left(a_{1}, b_{1}\right), \\ \frac{d}{d x}\left(\frac{\psi_{2}(x)}{\rho_{1}(x)}\right), & x \in\left(a_{2}, b_{2}\right), \\ \vdots & \\ \frac{d}{d x}\left(\frac{\psi_{n}(x)}{\rho_{1}(x)}\right), & x \in\left(a_{n}, b_{n}\right),\end{cases}
$$

where

$$
\begin{gathered}
\psi_{1}(x)=\frac{1}{\pi^{2}} \sum_{i=1}^{n} \int_{a_{i}}^{b_{i}}(-1)^{i} \frac{d}{d t}\left(f_{1}(t) \rho_{1}(t)\right) \ln \left|\frac{t-x}{t+x}\right| d t-\pi(-1)^{n} \sum_{i=1}^{2 n+1} A_{i} x^{(2 n+1-i)}, \\
\psi_{2}(x)=\frac{1}{\pi^{2}} \sum_{i=1}^{n} \int_{a_{i}}^{b_{i}}(-1)^{i+1} \frac{d}{d t}\left(f_{1}(t) \rho_{1}(t)\right) \ln \left|\frac{t-x}{t+x}\right| d t-\pi(-1)^{n} \sum_{i=1}^{2 n+1} A_{i} x^{(2 n+1-i)}, \\
\vdots \\
\psi_{n}(x)=\frac{1}{\pi^{2}(-1)^{n}} \sum_{i=1}^{n} \int_{a_{i}}^{b_{i}}(-1)^{i+1} \frac{d}{d t}\left(f_{1}(t) \rho_{1}(t)\right) \ln \left|\frac{t-x}{t+x}\right| d t+\pi \sum_{i=1}^{2 n+1} A_{i} x^{(2 n+1-i)} .
\end{gathered}
$$

We observe that the original integral equation (2.26) can be solved by using the above relations if and only if the following consistency conditions are satisfied:

$$
\psi_{1}\left(a_{i}\right)=\psi_{1}\left(b_{i}\right)=0, \quad i=1, \ldots, n .
$$

From these $2 n$ conditions, we find the arbitrary constant $\Pi_{i=2}^{2 n+1} A_{i}$, and from the order condition, the arbitrary constant $A_{1}$ can be determined, and finally the solution of the singular integral equation (2.26) can be obtained from (2.38).

In the next section, we give the genesis of the integral equation (2.2) with $B=(0, a) \cup$ $(b, c)$ and use its solution to the problem of scattering of water waves by a partially immersed vertical barrier with gap.

\section{Genesis of the Integral Equation (2.2) Connection with a Water Wave Problem}

\subsection{The Detailed Analysis and the Analytical Solution}

In this section, we show the connection of integral equation (2.2) with the problem of scattering of surface water waves by a thin partially immersed vertical barrier with a gap 


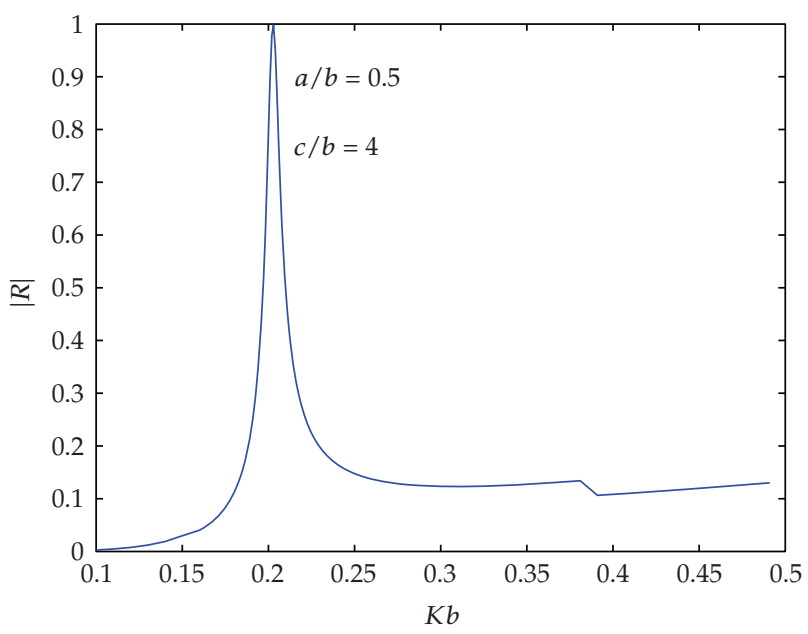

Figure 1

in deep water occupying the position $x=0,0<y<a$, and $b<y<c, y$-axis being directed downwards and $x$-axis along the mean free surface. We assume the motion to be two dimensional, irrotational, and time harmonic. The velocity potential is represented by $\operatorname{Re}(\phi(x, y) \exp (-i \sigma t)), \sigma$ being circular frequency. Here, $\phi(x, y)$ satisfies the following boundary value problem:

$$
\begin{gathered}
\nabla^{2} \phi=0 \quad \text { in } y \geq 0, \\
K \Phi+\phi_{y}=0 \quad \text { on } y=0,
\end{gathered}
$$

where $K=\sigma^{2} / g, g$ being the acceleration of gravity,

$$
\begin{gathered}
\phi_{x}=0 \quad \text { on } x=0, \quad y \in B \equiv(0, a) \cup(b, c), \\
r^{1 / 2} \nabla \phi \text { is bounded as } r \longrightarrow 0, \\
r=\left\{(x)^{2}+(y-v)^{2}\right\}^{1 / 2}, \quad v=a, b \text { or } c, \\
\nabla \phi \longrightarrow 0 \text { as } y \longrightarrow \infty, \\
\phi(x, y)= \begin{cases}R \exp (-K y-i K x)+\exp (-K y+i K x) & \text { as } x \longrightarrow-\infty, \\
T \exp (-K y+i K x) & \text { as } x \longrightarrow \infty,\end{cases}
\end{gathered}
$$




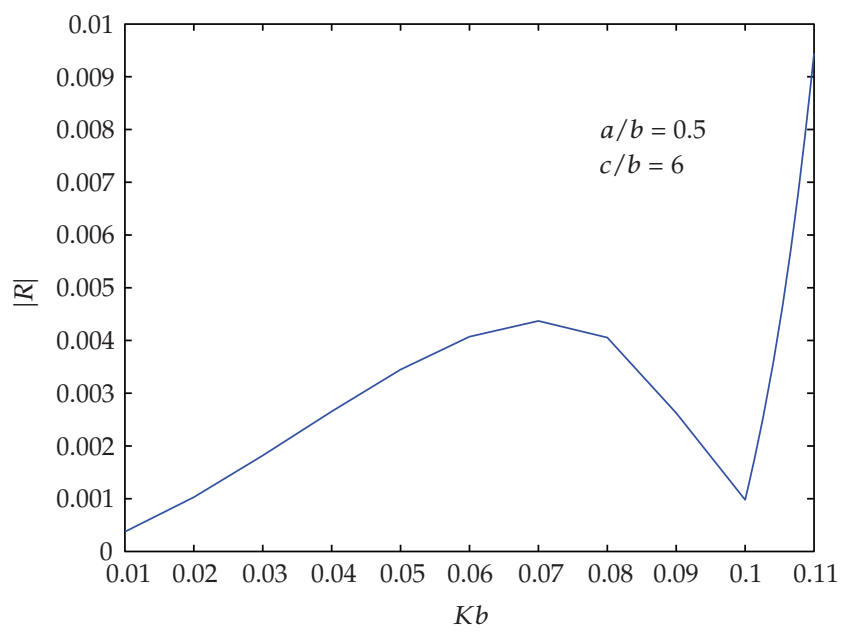

(a)

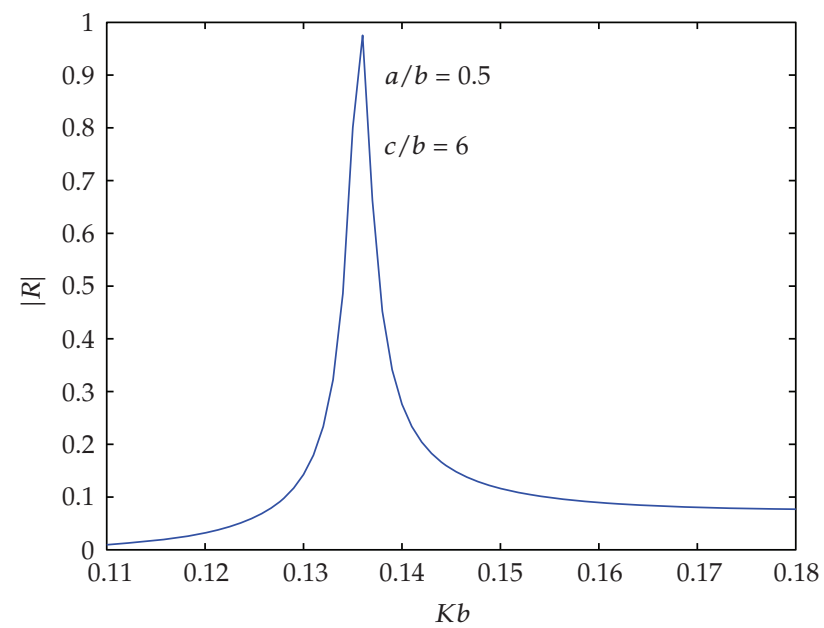

(b)

Figure 2

where $R$ and $T$ are reflection and transmission coefficients. A suitable representation of $\phi$ satisfying the equations (3.1), (3.4), and (3.5) is given by

$$
\phi(x, y)= \begin{cases}R \exp (-K y-i K x)+\exp (-K y+i K x)+\int_{0}^{\infty} B(k) L(k, y) \exp (k x) d k, & x<0, \\ T \exp (-K y+i K x)+\int_{0}^{\infty} A(k) L(k, y) \exp (-k x) d k, & x>0\end{cases}
$$

where,

$$
L(k, y)=k \cos k y-K \sin k y .
$$




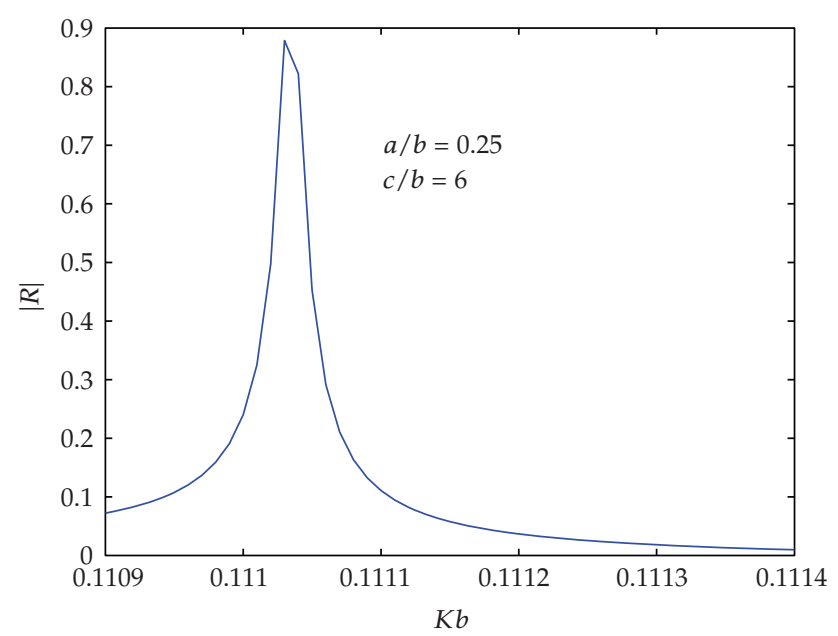

Figure 3

By Havelock's expansion theorem,

$$
A(k)=-B(k), \quad R=1-T .
$$

Then, using the condition (3.2) along with the condition of continuity of $\phi$ across the gap $G:(a, b) \cup(c, \infty)$, along $x=0$, we arrive at the following multiple integral equations:

$$
\begin{gathered}
\int_{0}^{\infty} k A(k) L(k, y) d k=i K(1-R) \exp (-K y), \quad y \in B, \\
\int_{0}^{\infty} A(k) L(k, y) d k=R \exp (-K y), \quad y \in G .
\end{gathered}
$$

The Relation (3.9) can be recast in the form

$$
\frac{d}{d y} \int_{0}^{\infty} \frac{A(k)}{k} L(k, y) d k= \begin{cases}i(1-R) \exp (-K y)+D_{1}, & 0<y<a \\ i(1-R) \exp (-K y)+D_{2}, & b<y<c\end{cases}
$$

where $D_{1}$ and $D_{2}$ are two arbitrary constants. Then, applying $d / d y+K$ to the equations (3.10) and (3.11), we obtain

$$
\int_{0}^{\infty} F(k) \sin k y d k=0, \quad y \in G,
$$


ISRN Applied Mathematics

$$
\int_{0}^{\infty} \frac{F(k)}{k} \sin k y d k= \begin{cases}K D_{1} y+C_{1}, & 0<y<a, \\ K D_{2} y+C_{2}, & b<y<c,\end{cases}
$$

where

$$
F(k)=-\left(k^{2}+K^{2}\right) A(k) .
$$

It is observed from the relation (3.13) that we must choose $C_{1}=0$ for consistency. Setting

$$
\int_{0}^{\infty} F(k) \sin k y d k=g(y), \quad y \in B
$$

where $g(y)$ is an unknown function and using sine inversion formula, to the relations (3.12) and (3.15), we obtain from (3.13)

$$
\int_{B} g(t) \log \left|\frac{y-t}{y+t}\right|=f(y), \quad y \in B
$$

with

$$
f(y)= \begin{cases}-\pi K D_{1} y, & 0<y<a, \\ -\pi K D_{2} y-\pi C_{2}, & b<y<c .\end{cases}
$$

The solution of the integral equation (3.16) is given by the equations (2.22), (2.23), and (2.24) with $f(y)$ given by relation (3.17).

Thus, knowing $g(t)$, we can obtain $F(k)$ by using Fourier Sine inversion formulae to the relations (3.12) and (3.15). Hence, $A(k)$ can be obtained from equation (3.14). Now, $f(y)$ contains unknown constants $D_{1}, D_{2}$, and $C_{2}$. To obtain the unknowns $D_{1}, D_{2}, C_{2}$, and $R$, we substitute $A(k)$ in (3.10) and (3.11) to get the following four equations:

$$
\begin{gathered}
\int_{b}^{c} \frac{\psi}{\rho} e^{K t} d t=0, \\
K\left[-\int_{0}^{a} \frac{\Psi}{\rho} e^{-K t} d t+\int_{b}^{c} \frac{\Psi}{\rho} e^{-K t} d t-\int_{0}^{a} \frac{\Psi}{\rho} e^{K t} d t\right]=R, \\
-K \int_{a}^{b} \frac{\Psi}{\rho} e^{K t} d t-K^{2} D_{1} \int_{0}^{a} x e^{K x}+e^{K b}\left(K b D_{2}-D_{2}+C_{2}\right)+D_{1}=0, \\
K \int_{a}^{b} \frac{\Psi}{\rho} e^{-K t} d t-K \int_{c}^{\infty} \frac{\psi}{\rho} e^{-K t} d t-\frac{K}{\pi} \int_{0}^{a}\left(-\pi K x D_{1}\right) e^{-K x} \\
-\frac{K}{\pi} \int_{b}^{c}\left(-\pi K x D_{2}-\pi C_{2}\right) e^{-K x}-D_{1}=i(1-R),
\end{gathered}
$$


where

$$
\begin{aligned}
\psi(x)= & -\frac{1}{\pi^{2}} \int_{0}^{a} \frac{d}{d t}(f(t) \rho(t)) \ln \left|\frac{t-x}{t+x}\right| d t+\frac{1}{\pi^{2}} \int_{b}^{c} \frac{d}{d t}(f(t) \rho(t)) \ln \left|\frac{t-x}{t+x}\right| d t \\
& -\pi\left(A x^{4}+B x^{3}+C x^{2}+D x+E\right)
\end{aligned}
$$

and $A, B, C, D$, and $E$ are arbitrary constants and

$$
\rho(x)=\left|x^{2}\left(x^{2}-a^{2}\right)\left(x^{2}-b^{2}\right)\left(x^{2}-c^{2}\right)\right|^{1 / 2}
$$

Thus, the relations (3.18) can be solved to determine four unknowns $D_{1}, D_{2}, C_{2}$, and $R$. This completes the description of the analytical solution of the water wave problem.

\subsection{Numerical Results}

The reflection coefficient $|R|$ has been numerically evaluated for various values of wave number $K b$ for four cases, namely, $a / b \rightarrow 0, c / b=15 ; a / b=0.5, c / b=4 ; a / b=0.5$, $c / b=6 ; a / b=0.25, c / b=6$.

Numerical computation of $|R|$ involves the solution of four equations (3.18). It may be noted from the relation (3.19) that for the solution of (3.18), one does not need to compute any integral with strong singularity. The integrals occurring in (3.18) are defined in Riemann sense. This is the main advantage in the present method as compared to the other methods based on strongly singular integral equation formulation available in the literature. Since there is no integral with strong singularity, so for numerical computation, we have used Mathematica 5.2 to evaluate various integrals in (3.18) and (3.19) and to solve the system of (3.18).

In Table 1 , we have presented the values of reflection coefficient $\left|R_{A}\right|$ computed from our result when $a / b \rightarrow 0$ and $c / b=15$ and $\left|R_{E}\right|$, the reflection coefficients corresponding to the problem of scattering by a submerged vertical barrier $x=0, b<y<c(c / b=15)$ as given in the literature (cf. Banerjea and Dutta [6]) for various values of wave number $K b$. It is observed that the values reflection coefficient $\left|R_{A}\right|$ coincides with $\left|R_{E}\right|$ upto two or three places of decimal. This shows that in limiting case, the reflection coefficient $\left|R_{A}\right|$ computed from the present paper agrees with the exact value of $\left|R_{E}\right|$ as given in [6].

In Figures 1, 2(a), 2(b), and $3(\mathrm{f}),|R|$ is depicted against $K b$ for various values of $a / b$ and $c / b=6$. In each case, we have verified that $|R|^{2}+|T|^{2}=1$ for all values of $K b$.

In Figure 1, we have plotted $|R|$ against various values of $K b$ when $a / b=0.5$ and $c / b=4$. For $0.15<K b<0.25$, it is observed that $|R|$ rises steeply, becomes almost a unity, and then falls rapidly forming a sharp peak. This indicates that for this range of values of $K b$, there occurs almost total reflection of wave energy. Also for $0.35<K b<0.4$, there occurs a small oscillation in $|R|$. In Figures 2(a) and 2(b), $|R|$ is depicted against $K b$ for $a / b=0.5$ and $c / b=6$. In Figure 2(a), it is observed that for very small values of $K b$, that is, for $K b<0.1,|R|$ increases at first and then decreases almost to zero indicating that most of the wave energy is transmitted. If the values of $K b$ are further increased, that is, $K b>0.1$, it is observed from Figure 2(b) that $|R|$ increases. For $0.13<K b<0.15,|R|$ sharply increases almost to unity and then decreases sharply. The peak value in this case is lower than the peak value when $c / b=4$ 
Table 1

\begin{tabular}{lcc}
\hline$K b$ & $\left|R_{E}\right|$ & $\left|R_{A}\right|$ \\
\hline 1.1 & 0.0870394 & 0.0704223 \\
1.2 & 0.0722664 & 0.0651027 \\
1.3 & 0.0599543 & 0.0620144 \\
1.4 & 0.0496994 & 0.0503388 \\
1.5 & 0.0411649 & 0.0408215 \\
1.6 & 0.0340685 & 0.0331023 \\
1.7 & 0.0281734 & 0.0268594 \\
1.8 & 0.0232811 & 0.0218163 \\
1.9 & 0.0192247 & 0.0177404 \\
2 & 0.0158647 & 0.0144455 \\
\hline
\end{tabular}

as observed in Figure 1. Thus, when the length of the lower part of the barrier increases, the peak value in $|R|$ decreases. In Figure $3,|R|$ is plotted against $K b$ for $a / b=0.25$ and $c / b=6$. In this figure, it is observed that when the length of the gap is increased keeping the length of the lower part of the barrier the same, the sharp peak in the values of $|R|$ occurs for $0.111<K b<0.1111$. In this case the peak value is much lower than the peak value observed in Figures 1 and 2(b). Thus, an increase in the length of the gap decreases the peak value in $|R|$. Thus, it is observed from Figures 1,2 , and 3 that for certain range of values of $K b$, there occurs a sharp peak in $|R|$ showing that $|R|$ reaches a maximum value. In these three cases, the maximum value $|R|$ is almost unity, that is, the incident wave experiences almost total reflection for certain range of small values of $K b$. This type of behaviour of $|R|$ is also observed by Banerjea in [9].

\section{Conclusion}

A direct function theoretic method is applied here to solve the integral equations (2.2) and (2.26) without converting them to the integral equation with strong singularity. It may be noted here that the solution functions given in (2.22) and (2.38) do not involve any strong singularity. Also using the presently detailed solution of (2.2), the problem of scattering of water waves by a partially immersed barrier with a gap is examined fully.

\section{Acknowledgments}

This work is also partially supported by DST through PURSE scheme (no. SR/S9/Z$23 / 2008 / 5$ ) at Jadavpur University. A. Chakrabarti is thankful to NASI, Allahabad for offering Senior Scientist Platinum Jubilee Fellowship which helped him in pursuing this research.

\section{References}

[1] L. Lewin, Theory of Wave Guides, Newness Butterworths, London, UK, 1975.

2. F. Ursell, "The effect of a fixed vertical barrier on surface waves in deep water," Proceedings of the Cambridge Philosophical Society, vol. 43, pp. 374-382, 1947.

[3] B. N. Mandal and P. K. Kundu, "Scattering of waterwaves by vertical barriers and associated Mathematical methods," Proceedings of the Indian National Science Academy, vol. 53, pp. 514-530, 1987. 
[4] A. Chakrabarti and S. R. Manam, "Solution of a logarithmic singular integral equation," Applied Mathematics Letters, vol. 16, no. 3, pp. 369-373, 2003.

[5] S. R. Manam, "A logarithmic singular integral equation over multiple intervals," Applied Mathematics Letters, vol. 16, no. 7, pp. 1031-1037, 2003.

[6] S. Banerjea and B. Dutta, "Solution of a singular integral equation and its application to water wave problems," Indian Institute of Science, vol. 86, no. 3, pp. 265-278, 2006.

[7] S. Banerjea and C. Sarkar, "On a singular integral equation with log kernel and its application," International Journal of Mathematics and Mathematical Sciences, vol. 2006, Article ID 35630, 12 pages, 2006.

[8] A. Chakrabarti, "Solution of certain weakly singular integral equations," IMA Journal of Applied Mathematics, vol. 71, no. 4, pp. 534-543, 2006.

[9] S. Banerjea, "Scattering of water waves by a vertical wall with gaps," Australian Mathematical Society, vol. 37, no. 4, pp. 512-529, 1996. 


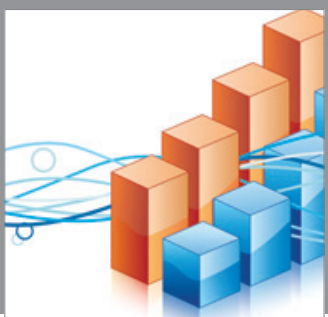

Advances in

Operations Research

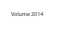

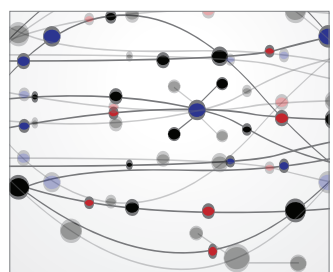

\section{The Scientific} World Journal
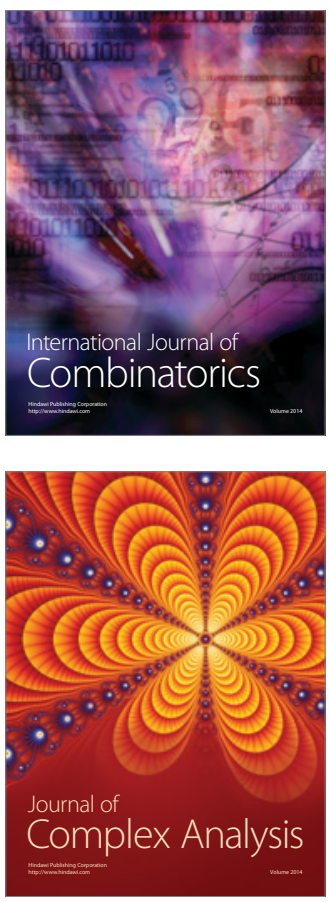

International Journal of

Mathematics and

Mathematical

Sciences
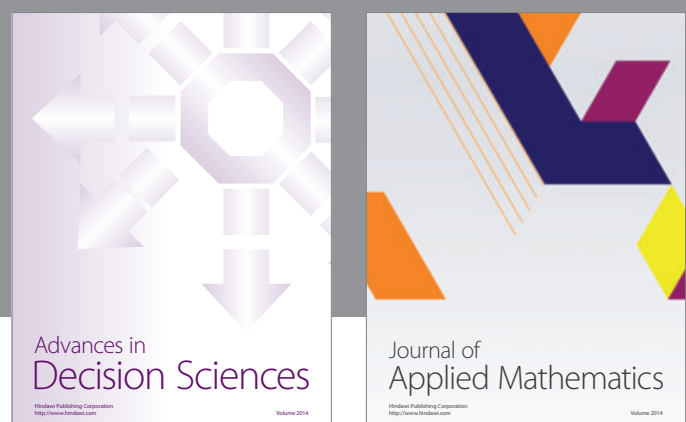

Journal of

Applied Mathematics
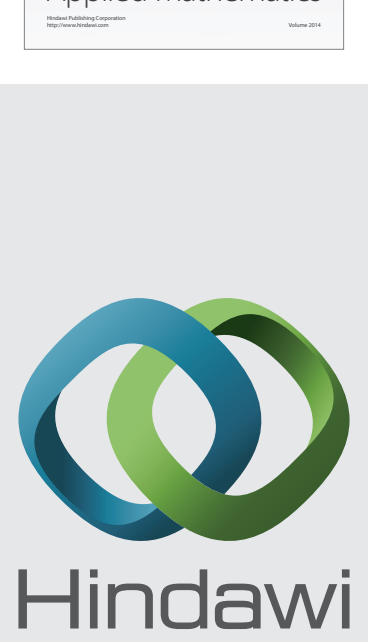

Submit your manuscripts at http://www.hindawi.com
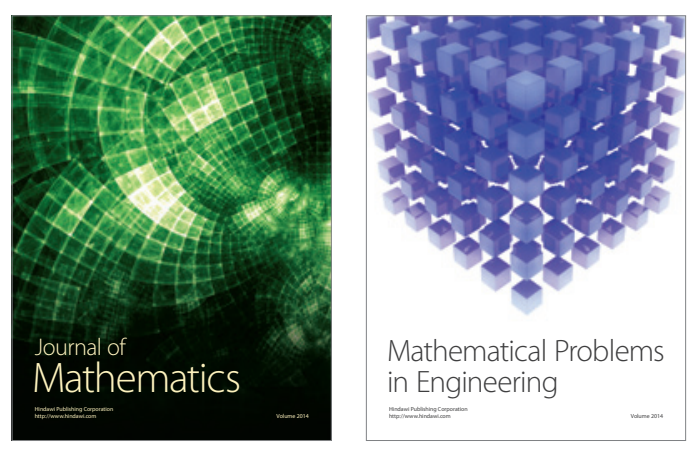

Mathematical Problems in Engineering
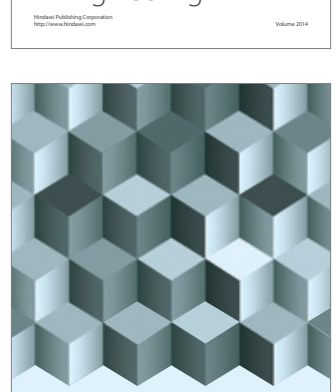

Journal of

Function Spaces
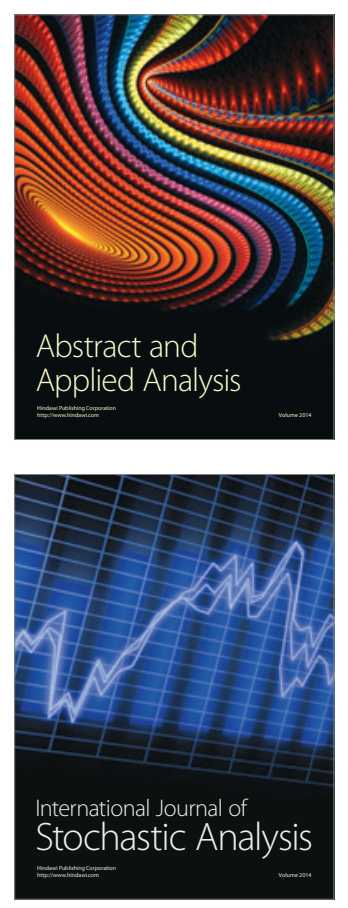

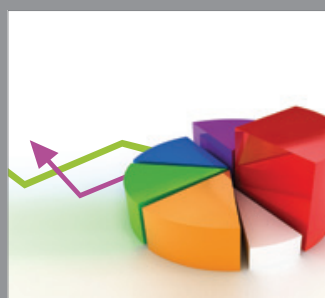

ournal of

Probability and Statistics

Promensencen
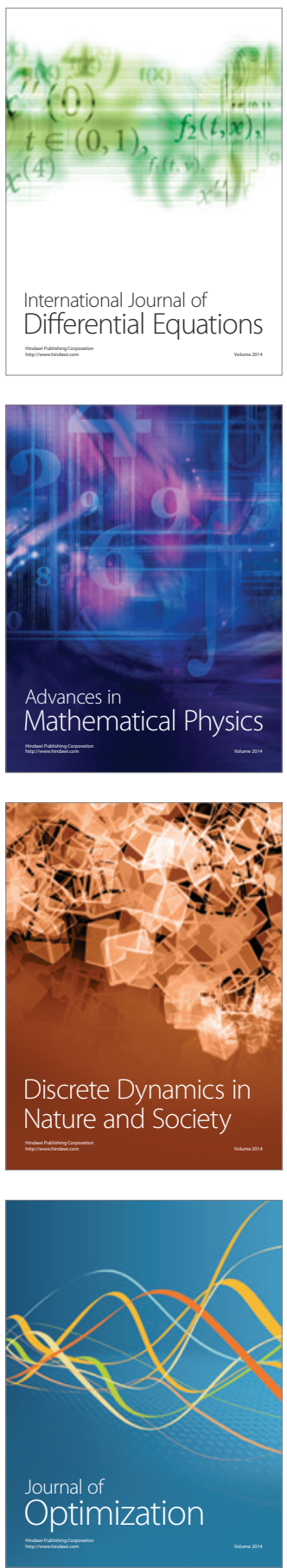